\title{
A Frequency-Domain Analytical Model of an Uncontrolled Single-Phase Voltage-Source Rectifier
}

\author{
Hamish D. Laird, Student Member, IEEE, Simon D. Round, Member, IEEE, and Richard M. Duke, Member, IEEE
}

\begin{abstract}
The harmonic currents generated by the single-phase rectifier are well known. As the levels of these currents become larger, the use of power conditioners such as shunt active filters to lower the levels is becoming more attractive. In order to analyze the interaction between the condition, ac system, and rectifier it is necessary to have an accurate model of the rectifier. This paper describes a frequency-domain analytical model of the single-phase rectifier. The model includes the dominant frequency transfer mechanisms. These are the direct transfer and that due to the modulation of the switching instants. A small-signal linearized analysis is presented and the behavior predicted is confirmed by perturbation analysis using time-domain simulation. Accurate results are obtained, and the importance of including the switching instant modulation is shown.
\end{abstract}

Index Terms-Frequency-domain analysis, harmonic analysis, power quality, rectifier.

\section{NOMENCLATURE}

$V_{S} \quad$ AC supply voltage.

$V_{\mathrm{AC}} \quad$ Fundamental supply voltage.

$\omega_{0} \quad$ Fundamental frequency.

$A$, Fundamental supply voltage magnitude.

$\Delta V \quad$ Perturbing voltage.

$V_{k} \quad$ Perturbing voltage magnitude.

$k \omega_{0} \quad$ Frequency of perturbing voltage.

$\delta_{k} \quad$ Perturbing voltage phase.

$\theta_{1} \quad$ Angle of turn-on switching instant.

$\theta_{2} \quad$ Angle of turn-off switching instant.

$V_{\text {DCBUS }}$ DC-bus voltage.

$V_{\mathrm{DC}} \quad$ Rectifier dc-side voltage.

$\Psi \quad$ Rectifier switching function Fourier series; used as rectifier frequency transfer function.

$\Psi_{\text {Simod }} \quad$ Switching modulation function Fourier series; used as switching modulation transfer function.

$t \quad$ Time.

$\omega_{0} t \quad$ Angular time.

\section{INTRODUCTION}

$\mathbf{S}$ INGLE-PHASE rectifiers and other nonlinear rectifier loads form a large part of present electrical load. The harmonic currents that these rectifiers produce flow in the

Manuscript received March 29, 1999; revised September 5, 1999. Abstract published on the Internet March 12, 2000.

The authors are with the Department of Electrical and Electronic Engineering, University of Canterbury, Christchurch, New Zealand (e-mail s.round@elec.canterbury.ac.nz).

Publisher Item Identifier S 0278-0046(00)04740-7. power system and can have detrimental effects [1], [2]. As the delivery of high-quality electrical power becomes increasingly important, the impact of interaction between loads and the power system needs to be characterized. Nonlinear loads pose a difficult problem, as the analysis of the interaction cannot use typical linear time-domain approaches such as the Laplace transform or linear time-invariant differential equations. However, recent research has shown that the current-source HV dc rectifier and other nonlinear power electronic switching devices are almost completely linear in the frequency domain [3]. The coupling between frequencies due to modulation can be described as a magnitude and phase change which can be accomplished by complex multiplication. This frequency-domain approach allows the nonlinear devices to be linearized and makes superposition valid [3].

The interaction of the single-phase rectifier with the system requires further investigation when the system includes power conditioning equipment such as a shunt active filter. Interactions such as that between a three-phase rectifier and shunt active filter can produce increased harmonic current flowing into the rectifier [4]. This is not the intention of the active filter. In order to determine the interaction of a single-phase rectifier with the ac system, with or without a power conditioner, an accurate model of the single-phase rectifier is required. Analysis of three-phase rectifier harmonics and system interaction has been performed [5], but the single-phase rectifier has received little attention. The interaction of power conditioning devices, the ac system, and power-factor-correction capacitors has been investigated [6], but the incorporation of accurate nonlinear load models has yet to be attempted.

This paper describes the development of a model of the single-phase rectifier using analytic frequency-domain techniques. There is the possibility of the single-phase rectifier having a phase-dependent response at characteristic harmonics as has been found with other switching converters [7]. This will have implications for active filtering, as a shunt active filter produces characteristic harmonics in the steady state. The determination of the response to frequencies other than harmonic frequencies gives the small-signal response and, hence, can be used to analyze the system's steady-state stability and transient response [8].

This paper first describes the operation of the rectifier in terms of base and switching instant modulation switching functions and their Fourier series. Suitable linearizations to obtain the switching instant variation are made and the total frequency transfer developed. 


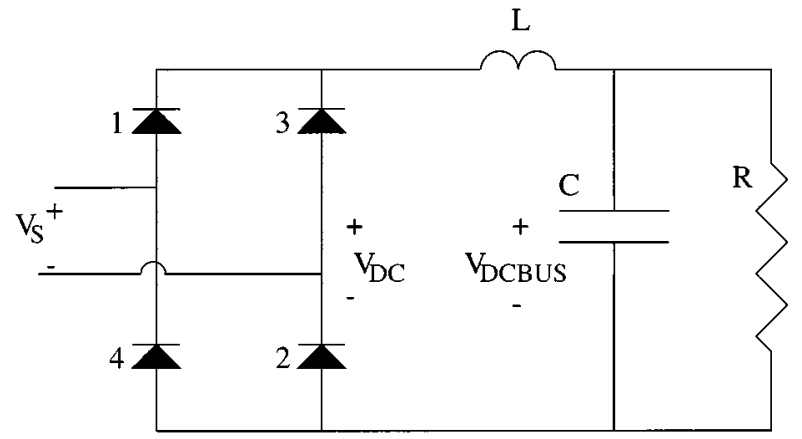

Fig. 1. Single-phase rectifier.

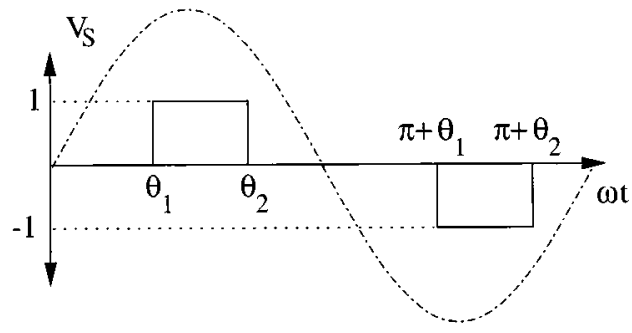

Fig. 2. Switching function of single-phase rectifier.

\section{FREQUENCY MODUlation}

The single-phase voltage-source rectifier operates as a modulator, since its primary function is to convert the fundamental power frequency $\omega_{0}(50$ or $60 \mathrm{~Hz})$ to dc $(0 \mathrm{~Hz})$. Modulation is achieved by the alternate switching action of diodes. As each diode is uncontrolled, the times at which the diodes switch (the switching instants or equivalent switching instant angles) are determined by the circuit conditions rather than by a switching control system such as that in a thyristor-controlled rectifier. The typical single-phase rectifier is shown in Fig. 1. Because of the desire for inexpensive construction, the rectifier typically operates in discontinuous current conduction mode and the value of the inductance $L$ is small.

\section{A. Base Switching Function}

The typical switching function $\Psi$ of the diode rectifier, shown in Fig. 2, represents the switching of the alternate diode pairs, (1 and 2 , and 3 and 4 , in Fig. 1), to connect the supply voltage $V_{S}$ to the dc-bus capacitor $C$ and the load $R$ through the inductor. The switching function shows diode pair 1 and 2's turn-on switching instant indicated by the angle $\theta_{1}$ and the turn-off switching instant, angle $\theta_{2}$. The switchings for diode pair 3 and 4 are in the voltage's $\mathrm{V}_{S}$ negative half cycle at angles $\pi+\theta_{1}$ and $\pi+\theta_{2}$. The switching function is given in Fourier series form in (1). This switching function operates as a frequency transfer function in that it describes the way an ac-side frequency is transferred to the dc side

$\Psi=\frac{4}{m \pi} \sin \left(\frac{m \theta_{1}-m \theta_{2}}{2}\right) \sin \left(m \omega_{0} t-\frac{m \theta_{1}+m \theta_{2}}{2}+\frac{\pi}{2}\right)$ for $m=1,3,5,7 \ldots$

$\Psi=0, \quad$ for $m=0,2,4,6 \cdots$

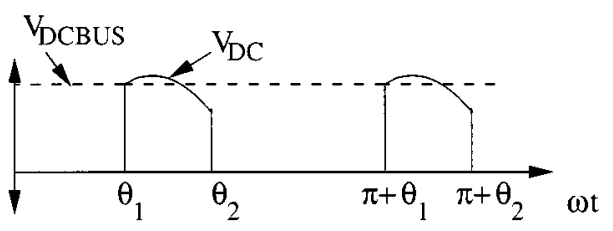

Fig. 3. DC-side voltage due to modulation from the ac voltage

The product of the switching function with an ac supply voltage of arbitrary frequency $k \omega_{0} t$ and phase $\delta_{k}$ gives the Fourier series for the resulting dc-side voltage $V_{\mathrm{DC}}$

$$
\begin{aligned}
V_{\mathrm{DC}}= & \frac{2}{m \pi} \sin \left[\frac{m \theta_{1}-m \theta_{2}}{2}\right] \\
& \cdot\left(\sin \left[(m+k) \omega_{0} t+\delta_{k}-\frac{m \theta_{1}+m \theta_{2}}{2}+\frac{\pi}{2}\right]\right. \\
& \left.\quad+\sin \left[(m-k) \omega_{0} t-\delta_{k}-\frac{m \theta_{1}+m \theta_{2}}{2}+\frac{\pi}{2}\right]\right),
\end{aligned}
$$

for $m=1,3,5,7 \ldots$

$V_{\mathrm{DC}}=0, \quad$ for $m=0,2,4,6 \cdots$

Fig. 3 shows the typical dc-side voltage waveform $V_{D C}$ for a fundamental $(k=1)$ applied voltage.

The pattern of frequency transfers described mathematically by (2) can be seen in the lattice diagram in Fig. 4 where for a given ac voltage input of frequency $k \omega_{0} t$ the frequencies of the resulting dc-side voltage $V_{\mathrm{DC}}$ can be determined. When a fundamental frequency, which corresponds to $k=1$, is applied, the resulting dc-side frequencies are $0 \mathrm{~Hz}, 100$ $\mathrm{Hz}, 200 \mathrm{~Hz} \cdots$. This is the normal operating condition of a single-phase rectifier and forms the base operating condition or unperturbed case for the following small-signal analysis. When a nonharmonic perturbing voltage, corresponding to $k$ having a noninteger value, is applied, the resultant dc-side frequencies are also noninteger multiples of the fundamental frequency and the corresponding frequencies, magnitudes, and phases are given by (2). As a graphical example, the dc-side frequencies that result from the $30-\mathrm{Hz}(k=0.6)$ component of an ac-side voltage made up of the base case fundamental voltage and a $30-\mathrm{Hz}$ perturbing voltage are shown by the dashed lines in Fig. 4. The dc-side frequencies are 20, 80, 120 , and $180 \mathrm{~Hz}$, corresponding alternately to $(m-k) \omega_{0} t$ and $(m+k) \omega_{0} t$ in (2).

\section{B. Switching Instant Modulation Spectrum}

Any ac-side supply voltage can change or modulate the turn-on and turn-off switching instants. The frequency components that arise from this switching instant modulation can be determined using the spectrum determined by Swcharz's analysis of the pulse duration modulator [9]. As an example the spectrum that results from the modulation 


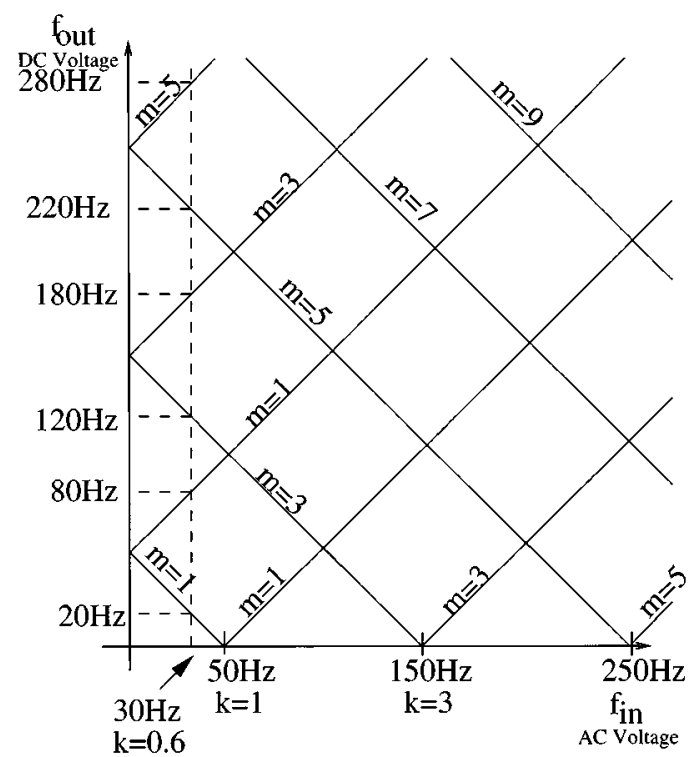

Fig. 4. DC-side voltage lattice diagram.

$\Delta \theta_{1}=b \cos \left(k \omega_{0} t+\delta_{k}\right)$ of the turn-on switching instant $\theta_{1}$ is given in (3) where $b$ is the magnitude of the variation

$$
\begin{aligned}
\Psi_{\text {Simod }}= & \frac{2}{m \pi}\left[J_{0}(m b)-1\right] \sin \left(m \omega_{0} t-m \theta_{1}\right) \\
& -\frac{2}{\pi} \sum_{n=1}^{\infty} \frac{J_{n}(m b)}{m} \\
& \cdot\left[\sin (m+n k) \omega_{0} t-m \theta_{1}+n \delta_{k}-\frac{n \pi}{2}\right] \\
& -\frac{2}{\pi} \sum_{n=1}^{\infty} \frac{J_{n}(m b)}{m} \\
& \cdot\left[\sin (m-n k) \omega_{0} t-m \theta_{1}+n \delta_{k}-\frac{n \pi}{2}\right], \\
& \text { for } m=1,3,5 \cdots \\
\Psi_{\text {Simod }}= & 0, \quad \text { for } m=2,4,6 \cdots .
\end{aligned}
$$

This spectrum gives a complete frequency-domain representation of the switching instant modulation which is used as a frequency transfer function in the same way as the rectifier switching function $\Psi$. The rectifier switching spectrum and the switching instant modulation spectrum completely characterize the behavior of the single-phase rectifier.

\section{Effect of Perturbing Voltage}

The single-phase rectifier is analyzed using a representative small perturbing voltage $\Delta V$, which is added to the fundamental supply voltage $V_{\mathrm{AC}}$. In the shunt active filter situation, the response to $\Delta V$ is the response of the single-phase rectifier to the small change in the supply voltage caused by the active filter current. The active filter will inject many harmonic current frequencies and so $\Delta V$ could have many frequencies. As the response of the rectifier is linearized, superposition can be used to evaluate all the frequencies individually.

The perturbing voltage $\Delta V$ has two effects in the singlephase rectifier. Firstly, $\Delta V$ is modulated by $\Psi$ to the dc side

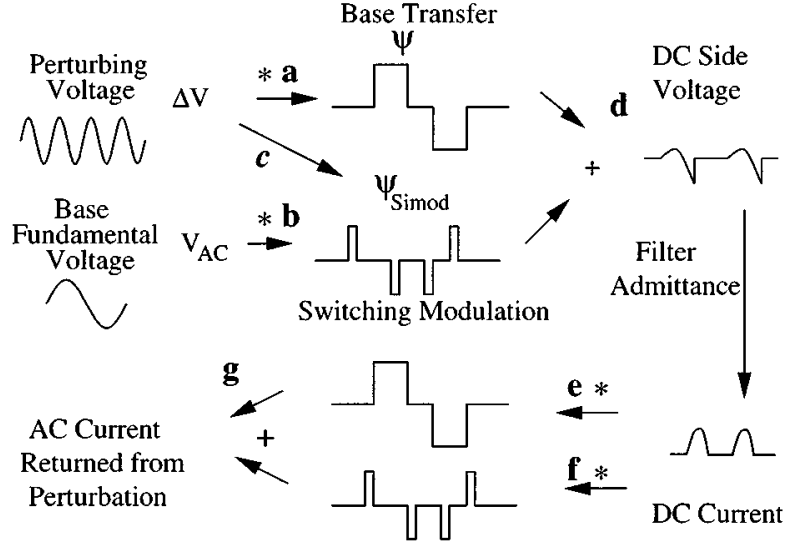

Fig. 5. Frequency transfer mechanism for the single-phase rectifier.

(shown by the arrow labeled $\mathbf{a}$ in Fig. 5 with the $*$ indicating a time-domain multiplication). That is, $\Delta V$ is multiplied by the base switching function and the result is a voltage on the dc side. Secondly, $\Delta V$ causes the changing of the switching instants or angles from their normal unperturbed or base operating values (c in Fig. 5.) The resultant switching instant modulation spectrum then forms the frequency transfer function $\Psi_{\text {Simod }}$. This then modulates $V_{\mathrm{AC}}$ (b in Fig. 5) in the same way as $\Psi$ modulates $\Delta V$. The two modulations, base and switching instant, characterize the response of the single-phase rectifier to $\Delta V$ and the sum of the two is the dc-side voltage (d in Fig. 5). The dc-side filter admittance converts this voltage to a current which is then remodulated back to the ac side by both $\Psi$ (e in Fig. 5) and $\Psi_{\text {Simod }}$ (f in Fig. 5). The ac-side current that results from the application of $\Delta V$ is the sum of the two effects (g in Fig. 5). In this analysis, it is assumed that $\Delta V$ is sufficiently small so that the system has essentially constant operating point and behaves linearly around this point. This assumption ensures that the two effects can be evaluated separately and combined by superposition. It is also assumed that the effect of $\Delta V$ modulated by the switching instant modulation $\Psi_{\text {Simod }}$ is negligible as both are small.

In summary, two effects make up the transfer of ac-side voltage to dc-side voltage and the transfer from dc-side current to ac-side current. These are the direct transfer by the base switching and that due to the switching instant modulation. As long as the modulation of the switching instants can be determined, these two switching transfers allow the behavior of the rectifier to be characterized.

\section{Switching Modulation Linearization}

The change in each switching instant angle is determined by linearizing the switching condition about the operating point. The conditions governing both the turn on and turn off switching instants are shown in Fig. 6. The turn-on switching occurs when $V_{S}$ exceeds $V_{\mathrm{DCBUS}}$, which is assumed constant for this analysis. The operating point is defined by the switching instant angles $\theta_{1}$ and $\theta_{2}$. The condition for the turn-on switching instant when $\Delta V$ is added to the ac voltage is

$$
V_{\mathrm{AC}}+\Delta V-V_{\mathrm{DCBUS}}=0
$$




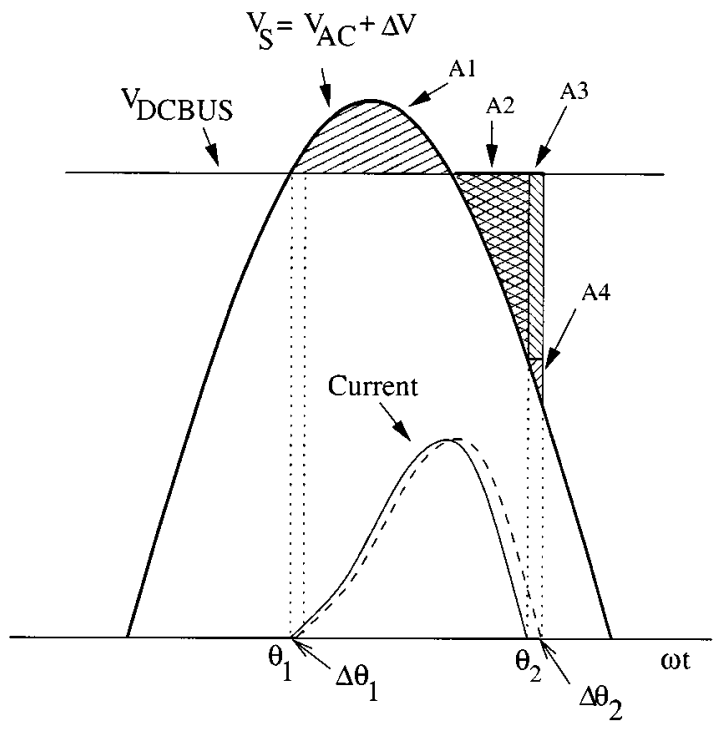

Fig. 6. Conditions to determine turn-on and turn-off switching instant modulations.

The base case fundamental ac voltage is

$$
V_{\mathrm{AC}}=A \sin \omega_{0} t .
$$

Rearranging (4) and substituting $\omega_{0} t=\theta_{1}$ gives

$$
A \sin \theta_{1}+\Delta V=V_{\mathrm{DC} \text { BUS }} .
$$

Letting $\theta_{1}=\theta_{1}+\Delta \theta_{1}$ and substituting this in (6) gives

$$
A \sin \left(\theta_{1}+\Delta \theta_{1}\right)+\Delta V=V_{\text {DCBUS }} \text {. }
$$

Upon expansion, the $\sin \left(\theta_{1}+\Delta \theta_{1}\right)$ becomes

$$
\sin \left(\theta_{1}+\Delta \theta_{1}\right)=\sin \theta_{1} \cos \Delta \theta_{1}+\cos \theta_{1} \sin \Delta \theta_{1} .
$$

Then, using the linearizing approximations

$$
\sin \Delta \theta_{1} \simeq \Delta \theta_{1}
$$

and

$$
\cos \Delta \theta_{1} \simeq 1
$$

that are valid as long as $\Delta \theta_{1}$ is sufficiently small (which is assumed to be the case for small applied $\Delta V$ ) gives

$$
A \sin \theta_{1}+A \cos \theta_{1} \Delta \theta_{1}+\Delta V=V_{\mathrm{DC} \text { BUS }} .
$$

At the switching instant

$$
V_{\mathrm{DCBUS}}=V_{\mathrm{AC}}=A \sin \theta_{1}
$$

which means that

$$
A \cos \theta_{1} \Delta \theta_{1}+\Delta V=0 .
$$

Rearranging (13) gives

$$
\Delta \theta_{1}=\frac{-\Delta V}{A \cos \theta_{1}}
$$

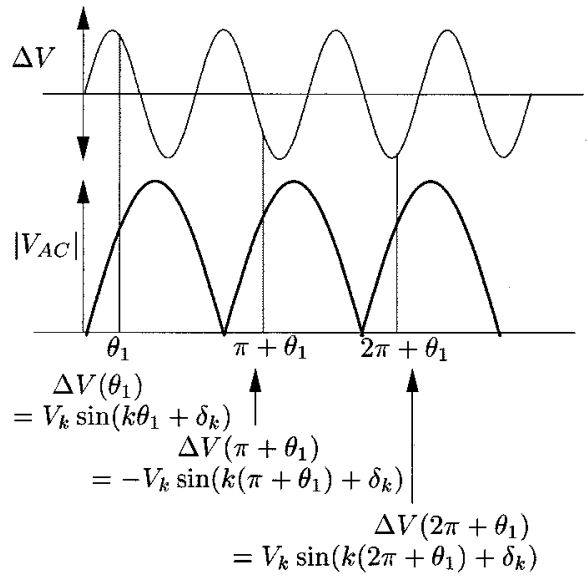

Fig. 7. Effective frequency of switching instant modulation.

which is the change $\Delta \theta_{1}$ in the turn-on switching instant, defined by the angle $\theta_{1}$, for a given perturbing voltage $\Delta V$ and fundamental magnitude $A$.

\section{E. Effective Frequency of Switching Instant Modulation}

The unperturbed switching of the single-phase rectifier occurs at twice the fundamental frequency. When a perturbing voltage $\Delta V=V_{k} \sin \left(k \omega_{0} t+\delta_{k}\right)$ is applied, the effect of that voltage on the switching instant modulation is determined by the interaction of the rectifier's twice fundamental switching and $\Delta V$. Fig. 7 shows how the switching action of the rectifier samples $\Delta V$ at twice the fundamental frequency. The value of $\Delta V$ that determines the switching instant variation is the value at each unperturbed switching instant. This value of $\Delta V$ at three successive switching instants is evaluated in Fig. 7 in order to find the frequency of the change in the switching instant angle. (The negative half cycle of the ac voltage is shown inverted and the sign of $\Delta V$ at the $\pi+\theta_{1}$ switching instant is negated for clarity. This is equivalent to actual rectifier operation.) As the sampling occurs at twice fundamental frequency, a modulation or frequency shift occurs, meaning that the effective switching instant modulation frequency is different from the frequency of $\Delta V$. In order to determine this frequency, the values of $\Delta V$ from (15) are shown for successive turn-on switching instants in (16)-(18)

$$
\Delta V=V_{k} \sin \left(k \omega_{0} t+\delta_{k}\right)
$$

for $\omega_{0} t=\theta_{1}$

$$
\Delta V=V_{k} \sin \left(k \theta_{1}+\delta_{k}\right)
$$

for $\omega_{0} t=\pi+\theta_{1}$

$$
\Delta V=V_{k} \sin \left(k \theta_{1}+\delta_{k}+k \pi \pm \pi\right)
$$

for $\omega_{0} t=2 \pi+\theta_{1}$

$$
\Delta V=V_{k} \sin \left(k \theta_{1}+\delta_{k}+2 k \pi\right) .
$$




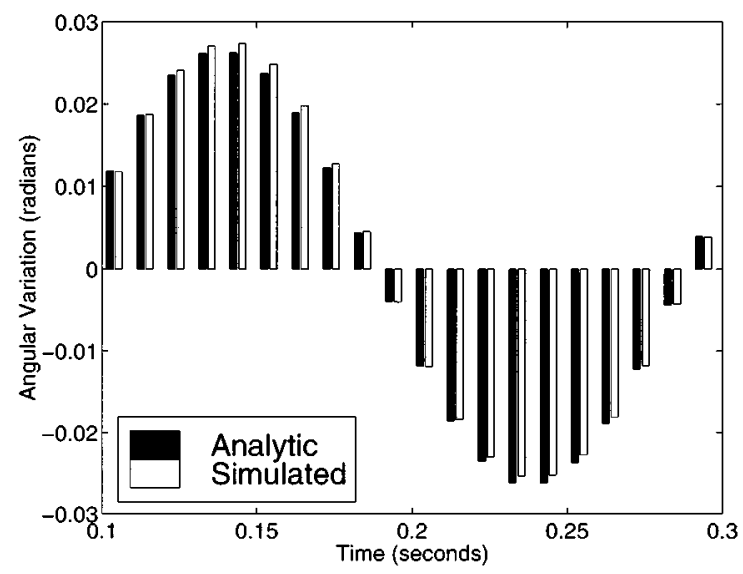

Fig. 8. Turn-on switching instant modulation validation.

Any function that produces these values at these switching instants is a valid effective switching instant modulation. One such function is

$$
\Delta V_{\text {effective }}=V_{k} \sin \left((k-1) \omega_{0} t+\theta_{1}+\delta_{k}\right)
$$

This means that a perturbing voltage $\Delta V$ of frequency $k \omega_{0}$ affects the switching instant at a frequency $(k-1) \omega_{0}$. However, as this result is from possibly sub-Nyquist sampling (which is the situation shown in Fig. 7) other expressions that produce a valid $\Delta V_{\text {effective }}$ can be developed. The choice of the effective frequency is arbitrary because an effective switching instant modulation can be found involving frequency $(k-3) \omega_{0},(k-5) \omega_{0}$, $(k+1) \omega_{0}$, or any other frequency an odd multiple of $\omega_{0}$ different to $k$. Each of these functions generates the same value for the switching instant modulation at each switching instant. That is, when the switching instant modulation is used in the switching instant modulation spectrum (3), the resultant frequency components are the same. The $(k-1) \omega_{0}$ function is used for calculation purposes in this paper.

\section{F. Switching Instant Modulation Validation}

The switching instant modulation caused by the applied voltage $\Delta V$ was measured using a time-domain Matlab/Simulink simulation of the rectifier. A $0.5 \%$ sinusoidal voltage $\left(V_{k}=1.5 \mathrm{~V}\right)$ of frequency $45 \mathrm{~Hz}$ and phase $\delta_{k}=1.3708$ rad was added to the $50-\mathrm{Hz} 230-\mathrm{Vrms}$ fundamental voltage. The dc-bus voltage is $320 \mathrm{Vdc}, \theta_{1}=1.3906$, and $\theta_{2}=1.9316$, which is a reasonable approximation to a typical single-phase rectifier. The effect of $\Delta V$ on the turn-on switching instants is shown in Fig. 8 by the light bars. As can be seen, these are well approximated by the dark bars which are calculated using (19) and (14). Each angular variation occurs at the same time instant but they are shown side by side in Fig. 8 for clarity. The mean absolute error in the switching instants is $4 \%$ for this case. Similarly accurate results are obtained for other perturbing frequencies.

\section{G. Turn-Off Switching Instant Modulation}

The turn-off switching instant modulation is determined by equating the integral of the voltage applied to the dc-side inductor to zero. That is, the dc-side current returns to zero, the condition for the switch off, when the total integral volt seconds is zero. Fig. 6 shows the ac-side and dc-side voltages that determine this switching. The integral of the voltage across the inductor is

$$
\int_{\theta_{1}}^{\theta_{2}+\Delta \theta_{2}}\left(V_{\mathrm{AC}}+\Delta V-V_{\mathrm{DCBUS}}\right) d \theta=0 .
$$

The modulation of $\theta_{2}$ does not depend on the modulation of $\theta_{1}$, as the contribution to the integral from the modulation of $\theta_{1}$ is negligibly small. It is, therefore, ignored. By separating the contributions in (20) and rearranging the limits, the individual effects can be found

$$
\begin{gathered}
\int_{\theta_{1}}^{\theta_{2}}\left(V_{\mathrm{AC}}-V_{\mathrm{DCBUS}}\right) d \theta+ \\
\int_{\theta_{2}}^{\theta_{2}+\Delta \theta_{2}} \Delta V d \theta+ \\
\int_{\theta_{2}}^{\theta_{2}+\Delta \theta_{2}}\left(V_{\mathrm{AC}}-V_{\mathrm{DCBUS}}\right) d \theta+ \\
\int_{\theta_{1}}^{\theta_{2}} \Delta V d \theta=0 .
\end{gathered}
$$

The applied perturbing voltage $\Delta V$ is

$$
\Delta V=V_{k} \sin \left(k \omega_{0} t+\delta_{k}\right)
$$

The ac fundamental voltage is

$$
V_{\mathrm{AC}}=A \sin \omega_{0} t
$$

and the dc-bus voltage is

$$
V_{\mathrm{DC} B U S}=A \sin \theta_{1} .
$$

The term from (21), which corresponds to areas A1 and A2 in Fig. 6, is zero, as this is the situation that determines the turn-off switching instant in the case where no $\Delta V$ is applied (the base case). The effect of $\Delta V$ in the interval from $\theta_{2}$ to $\theta_{2}+\Delta \theta_{2}$ is

$$
\begin{aligned}
\int_{\theta_{2}}^{\theta_{2}+\Delta \theta_{2}} \Delta V d \theta= & {\left[\frac{-V_{k}}{k} \cos \left(k \theta+\delta_{k}\right)\right]_{\theta_{2}}^{\theta_{2}+\Delta \theta_{2}} } \\
= & \frac{-V_{k}}{k} \sin \left(k \theta_{2}+\delta_{k}\right) \sin k \Delta \theta_{2} \\
& +\frac{-V_{k}}{k} \cos \left(k \theta_{2}+\delta_{k}\right) \cos k \Delta \theta_{2} \\
& -\frac{-V_{k}}{k} \cos \left(k \theta_{2}+\delta_{k}\right) .
\end{aligned}
$$

As long as $k \Delta \theta_{2}$ is sufficiently small, the two cosine terms add to zero. The $\left(\sin k \Delta \theta_{2}\right) /(k)$ contribution is proportional to $\Delta \theta_{2}$ when $\Delta \theta_{2} \rightarrow 0$. This means that the contribution of this integral can be ignored if the other terms contributing to the total integral are significantly larger than $\Delta \theta_{2}$. The effect of $V_{\mathrm{AC}}$ in the interval $\theta_{2}$ to $\theta_{2}+\Delta \theta_{2}$ (23), which corresponds to areas A3 and $\mathrm{A} 4$ in Fig. 6, is

$$
\begin{aligned}
\int_{\theta_{2}}^{\theta_{2}+\Delta \theta_{2}}\left(V_{\mathrm{AC}}-V_{\mathrm{DC} B U S}\right) & d \theta \\
= & {\left[-A \cos \theta-A \theta \sin \theta_{1}\right]_{\theta_{2}}^{\theta_{2}+\Delta \theta_{2}} . }
\end{aligned}
$$




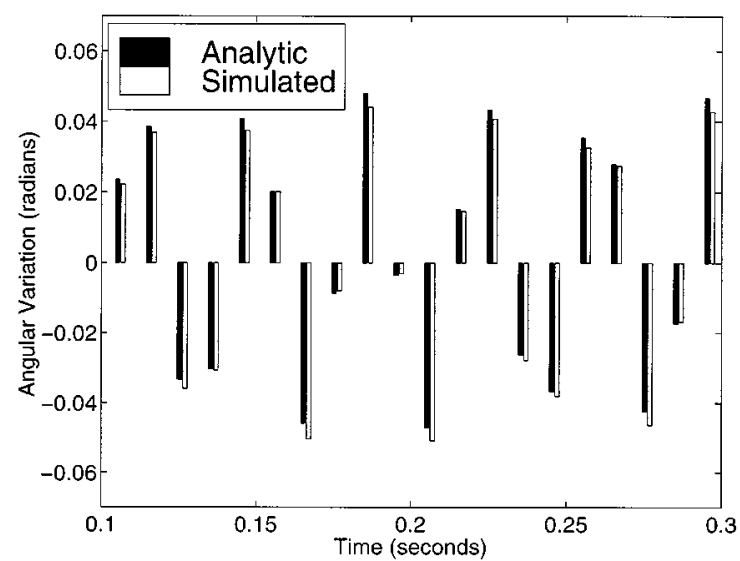

Fig. 9. Turn-off switching instant modulation validation

By grouping the terms and making the small value approximations (9) and (10), the contribution of this term is

$$
\Delta \theta_{2} A\left(\sin \theta_{2}-\sin \theta_{1}\right)
$$

The final term (22) is the effect of $\Delta V$ during the interval $\theta_{1}$ to $\theta_{2}$

$$
\begin{aligned}
\int_{\theta_{1}}^{\theta_{2}} \Delta V d \theta & =\left[-\frac{V_{k}}{k} \cos \left(k \theta+\delta_{k}\right)\right]_{\theta_{1}}^{\theta_{2}} \\
& =\frac{V_{k}}{k}\left[\cos \left(k \theta_{1}+\delta_{k}\right)-\cos \left(k \theta_{2}+\delta_{k}\right)\right] .
\end{aligned}
$$

Attheturn-off switchinginstant $\omega_{0} t=\theta_{2}$ and $\theta_{1}=\omega_{0} t-\theta_{2}+\theta_{1}$, so substituting for $\theta_{1}$ and $\theta_{2}$ then simplifying gives

$$
\frac{2 V_{k}}{k} \sin \left(k \omega_{0} t+\delta_{k}-\frac{k \theta_{2}}{2}+\frac{k \theta_{1}}{2}\right) \sin \left(\frac{k \theta_{1}}{2}-\frac{k \theta_{2}}{2}\right) .
$$

Using the same sampling approach as previously described in (15)-(18) gives the effective frequency and phase of the turn-off switching instant modulation due to applied $\Delta V(25)$. By then taking the two nonzero integral results (30) and the sampled (32) and making their sum zero, the turn-off switching instant modulation $\Delta \theta_{2}$ is

$$
\begin{aligned}
\Delta \theta_{2}= & \frac{2 V_{k} \sin \left(\frac{k \theta_{1}-k \theta_{2}}{2}\right)}{k A\left(\sin \theta_{2}-\sin \theta_{1}\right)} \\
& \cdot \sin \left((k-1) \omega_{o} t+\theta_{2}+\delta_{k}+\frac{k \theta_{1}-k \theta_{2}}{2}\right) .
\end{aligned}
$$

Note that $\Delta \theta_{2}$ does not depend on the inductance value $L$ except through the dependence of the operating points $\theta_{1}$ and $\theta_{2}$ on the inductance. Validation of the analysis is shown in Fig. 9 with the simulated variation (lighter bars) matching those calculated (darker) with (33). The mean absolute error is $4 \%$. The operating condition is the same as that for the previous turn-on switching instant example in Fig. 8. The frequency of the perturbing voltage is $123 \mathrm{~Hz}$ at phase $\delta_{k}=\pi$ and the magnitude $V_{k}=1.5$. ( $V_{k} \simeq 0.5 \%$ of the fundamental magnitude.)

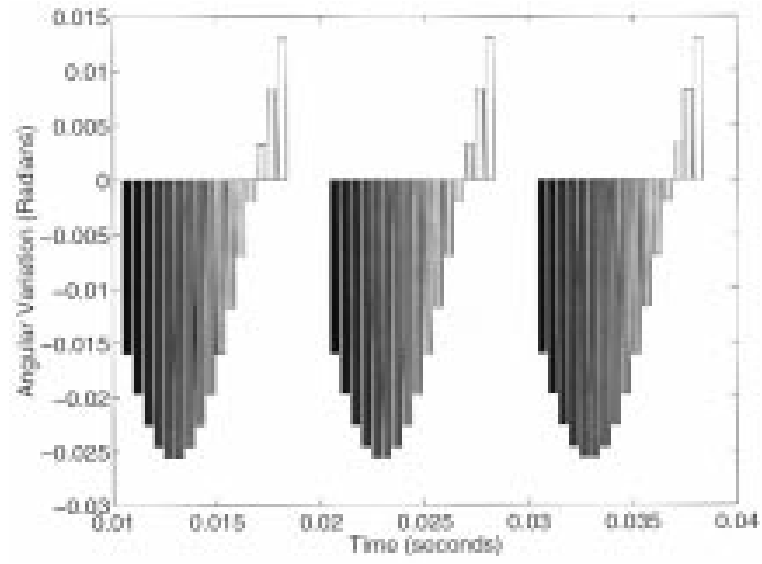

Fig. 10. Phase dependency of front switching instant modulation at fifth harmonic frequency, $250 \mathrm{~Hz}$. (Darkest bar: $\delta_{k}=0$; lightest bar: $\delta_{k}=\pi$.)

\section{FREQUENCY TRANSFER COMPARISON}

Once the operating point is determined, the base case transfer function $\Psi$ is set. Repeated calculation of different frequency transfers involves only the recalculation of the switching instant modulation spectra $\Psi_{\text {Simod }}$ and the convolution of the spectra. It was found that, if the applied frequency is a noninteger multiple of the fundamental frequency, accurate results can be found using only the first-order dc-side frequencies from the switching instant modulation spectrum. This corresponds to calculating only the $n=1$ terms in (3). This allows the switching modulation frequency transfer to be quickly calculated. When harmonic frequencies (both noncharacteristic and characteristic) are applied, it is necessary to calculate a number of higher order, $n=2,3,4, \cdots$, terms from the switching instant modulation spectrum equation (3), as these terms produce the same frequencies as the first-order $n=1$ terms. If a perturbing voltage that is a characteristic harmonic voltage, $\Delta V=V_{k} \sin \left(k \omega_{0} t+\delta_{k}\right)$, $k=1,3,5, \cdots$, is applied, the switching instant modulation is a constant zero-frequency value for both the turn-on and the turn-off switching instant. This strongly couples any applied characteristic harmonic voltage to the dc-side voltage at zero frequency. As the dc-side admittance is very high at low frequencies, this coupling of the characteristic harmonic to zerofrequency dc-side voltage produces a very large effect on the dc current. It may be that this coupling is sufficient to change the operating point defined by $\theta_{1}$ and $\theta_{2}$. This is not investigated in this paper, but will require further investigation. The switching instant modulation effect is strongly dependent on the phase $\delta_{k}$ of $\Delta V$ at characteristic harmonic frequencies. Fig. 10 shows the turn-on switching instant modulation that occurs when a fifth harmonic $(250 \mathrm{~Hz})$ perturbing voltage is applied with a phase $\delta_{k}$ which varies from 0 (darkest bar) to $\pi$ (lightest bar). (As previously, the switchings are coincident but are shown side by side for clarity.) The modulation is constant at each switching for the same phase, but different phases produce different amplitudes of switching modulation. At a certain phase, the characteristic harmonic perturbing voltage does not modulate the switching instant as the perturbing voltage is zero at each switching instant. As a result, the single-phase rectifier exhibits a phase-dependent response at characteristic harmonic frequencies. 

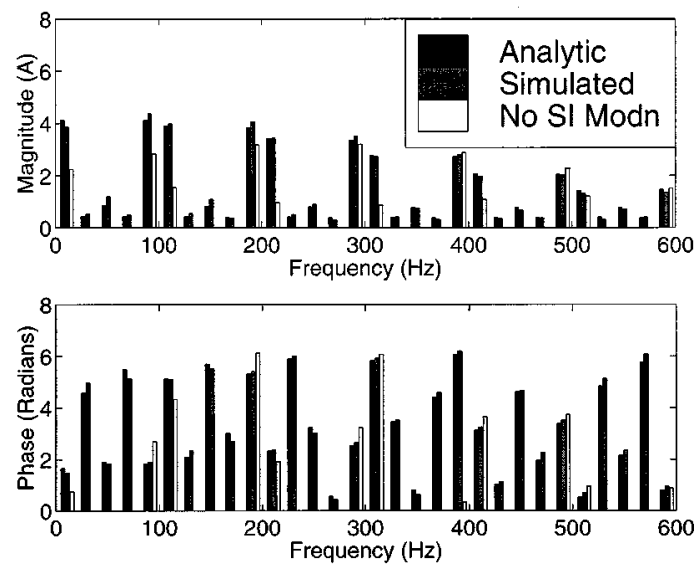

Fig. 11. Frequency transfer comparison—applied ac voltage to ac current. Analytic (dark bars); time-domain simulated (gray bars); no switching instant modulation (No SI Modn-white bars).

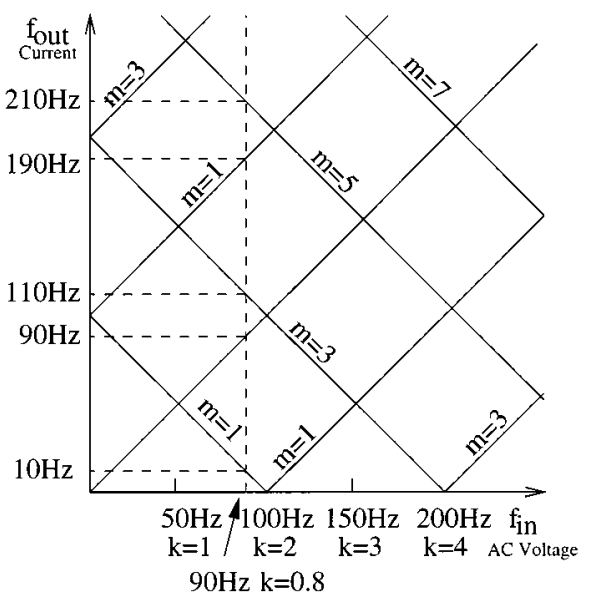

Fig. 12. AC voltage to ac current transfer lattice

In order to validate the frequency-domain analysis of the singlephase rectifier, a time-domain simulation was performed using Matlab/Simulink. The rectifier was simulated with only the fundamental supply voltage applied to form the base case. Then, the system was simulated again with a perturbing voltage $\Delta V$ added to the fundamental supply voltage. The difference between the two results is the response to the applied $\Delta V$. Fig. 11 shows the ac-side current that results from an applied voltage $\Delta V$ of magnitude $3 \mathrm{~V}$, frequency $90 \mathrm{~Hz}$, and phase 0 for both the time-domain simulation (gray bars) and the frequency-domain model (dark bars). The match is very good in both the magnitude and phase with the mean error $4 \%$. Also shown by the white bars is the result from the frequency domain model if the switching instant modulation is ignored. The accuracy of this approach is lower.

The smaller terms at frequencies $30 \mathrm{~Hz}, 50 \mathrm{~Hz}, 70 \mathrm{~Hz}, 130 \mathrm{~Hz}$, $150 \mathrm{~Hz} \cdots$ are due to second-order effects, meaning that they are the result of convolution of two first-order frequency components. They match the time-domain simulation results relatively well, even though it was not the intention to produce a model accurate in the second-order frequency products in this example. The analysis ignoring the switching instant modulation (white bars in Fig. 11) does not produce these terms. The first-order frequencies present in the ac current (those with the large magnitudes at 10 $\mathrm{Hz}, 90 \mathrm{~Hz}, 110 \mathrm{~Hz}, 190 \mathrm{~Hz} \cdots$ ) are as shown in Fig. 12. This lattice diagram shows, for clarity, only the first-order frequency couplings, but can be extended to include the second-order and other higher order couplings. The lattice can represent the full matrix of frequency couplings, including the switching instant modulation effects. It can, therefore, be used to determine the interaction of the rectifier with the ac system [10].

\section{CONCLUSIONS}

This paper has presented a frequency-domain analytical model of the single-phase voltage-source rectifier. The accuracy of the analysis and model has been confirmed by comparison of results to those obtained from time-domain simulation. The two effects that dominate the behavior of the rectifier are the transfer of any applied voltage to the dc side by the base switching function and the transfer of frequencies to the dc side due to the modulation of the switching instants. The switching instant modulations have been determined by linearizing the switching conditions, and this approximation has proved suitably accurate. The phase dependency of the switching instant modulation at characteristic harmonics was demonstrated. Ignoring the effect of switching instant modulation makes the model inaccurate. The rectifier model provides complete characterization of the methods and behavior of the modulation of frequency components by the rectifier. Such understanding of the mechanisms allows the interaction of the single-phase rectifier with the ac system to be determined. This means that the implications of design choices on the power quality effects of the rectifier itself and any power conditioning equipment installed with the rectifier can be found. By using the frequency domain, it will be possible to include the characteristics of the rectifier into the design considerations of any power conditioner control system.

\section{REFERENCES}

[1] G. T. Heydt, Electric Power Quality. West Lafayette, IN: Stars in a Circle, 1991.

[2] J. Arrillaga, D. A. Bradley, and P. S. Bodger, Power System Harmonics. Chichester, U. K.: Wiley, 1985.

[3] A. R. Wood and J. Arrillaga, "HVDC convertor waveform distortion: A frequency domain approach," Proc. IEE-Generation, Transmission Distribution, vol. 142, no. 1, pp. 88-96, 1996.

[4] F. Z. Peng and J. S. Lai, "Application considerations and compensation characteristics of shunt active and series active filters in power systems," in Proc. Int. Conf. Harmonics and Quality of Power, Las Vegas, NV, 1996, pp. 12-20.

[5] M. Sakui and H. Fujita, "An analytic method for calculating harmonic currents of a three-phase diode bridge rectifier with dc filter," IEEE Trans. Power Electron., vol. 9, pp. 631-637, Nov. 1994.

[6] H. Akagi, "Control strategy and site selection of a shunt active filter for damping of harmonic propagation in power systems," IEEE Trans. Power Delivery, vol. 12, pp. 354-363, Jan. 1997.

[7] B. C. Smith, N. R. Watson, A. R. Wood, and J. Arrillaga, "Harmonic tensor linearization of HVDC converters," IEEE Trans. Power Delivery, vol. 13, pp. 1244-1250, Oct. 1998.

[8] S. Todd, A. R. Wood, and P. S. Bodger, "An $s$-domain model of an HVDC converter," IEEE Trans. Power Delivery, vol. 12, pp. 1723-1729, Oct. 1997.

[9] M. Swcharz, W. R. Bennet, and S. Stein, Communications Systems and Techniques. New York: McGraw-Hill, 1966.

[10] E. V. Larsen, D. H. Baker, and J. C. McIver, "Low order harmonic interaction on AC/DC systems," IEEE Trans. Power Delivery, vol. 4, pp. 493-501, Jan. 1989. 


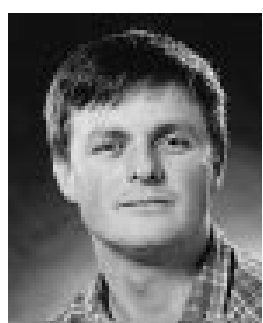

Hamish D. Laird (S'98) received the B.E. (Hons.) and Masters degrees in electrical and electronic engineering in 1991 and 1992, respectively, from the University of Canterbury, Christchurch, New Zealand, where he is currently working toward the Ph.D. degree.

Following receipt of the Masters degree, he worked in New Zealand on induction motor drives and starters. He gained experience with $\mathrm{HV}$ dc transmission systems and reactive power control in the U.K., and his most recent work was with a motor drives company where he designed and developed dc and ac drives. His research interests are power electronics and active filters and their effects on power quality.

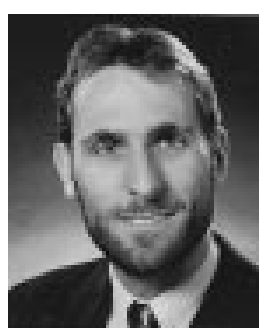

Simon D. Round (S'87-M'89) received the B.E. (Hons.) and Ph.D. degrees from the University of Canterbury, Christchurch, New Zealand, in 1989 and 1993, respectively.

He has held the positions of Research Associate in the Department of Electrical Engineering, University of Minnesota, and Research Fellow at the Norwegian Institute of Technology, Trondheim, Norway. Since 1995, he has been a Lecturer in the Department of Electrical and Electronic Engineering, University of Canterbury. His current research interests are in the areas of power quality and active power conditioners and electric vehicles.

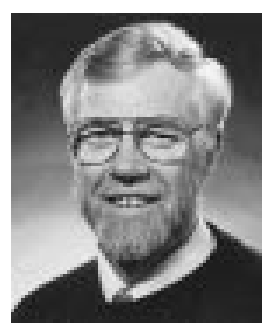

Richard M. Duke (S'75-M'79) received the B.Sc. degree in mathematics and the B.E. (Hons.), M.E., and $\mathrm{Ph} . \mathrm{D}$. degrees in electrical engineering from the University of Canterbury, Christchurch, New Zealand, in 1965, 1973, 1976, and 1979, respectively.

$\mathrm{He}$ was an Engineer with the New Zealand Electricity Department. Since 1984, he has held a lecturing position at the University of Canterbury, where he is currently the Head of the Department of Electrical and Electronic Engineering. His current research interests are in the area of power quality and active filtering for power systems applications and low-voltage high-current-switching power supplies for industrial applications.

Dr. Duke is a Past Chairman of the New Zealand South Section of the IEEE and a Registered Professional Engineer in New Zealand. 